\title{
To Reduce Chemical Consumption in Pretreatment Process of Terry Towel Processing
}

\author{
Kiran Patil, Tushar A Shinde* and Raichurkar PP \\ Mukesh Patel School of Technology, SVKM'S NMIMS, India
}

*Corresponding author: Tushar A Shinde, SVKMS, NMIMS, Centre for Textile Functions, Mukesh Patel School of Technology, Management Shirpur, District Dhule-425405, India

submission: 㘹August 01, 2018 Published: 眥 August 14, 2018

\begin{abstract}
This study is aim to standardize the process parameter is desizing, scouring and bleaching and come out with the best practice in chemical consumption in pretreatment of terry towel. The determination of the pretreatment performance because of desizing, scouring and bleaching chemical concentration.

The careful consideration of process parameter in pretreatment helps the wet processing industry to ensure the application of standard concentration of the chemical during processing. The concentration of alkali use in the pretreatment of cotton is represented by many researches.

In this process by varying the chemical concentration, time and temperature, we obtain good result. This particular research on the reduction of the total chemical consumptions, time and temperature. The results were found to promising and comparable to that conventional pretreatment fabric which is performing in terry towel industry.

The aim of this project work is to develop less consumption of chemical pretreatment method which can produce similar desizing efficiency, whiteness index, and absorbency of the fabric. In this study we found reduction of pollution load associated with excessive usage of chemical in standard conventional pretreatment process.
\end{abstract}

It was found that with the reduction of caustic, stabilizer and Hydrogen Peroxide concentration performance of pretreatment become better. Effect of the above process by reducing chemical consumptions in desizing scouring, bleaching (pretreatment) of terry towel fabric in soft-flow Thies machine which gives good results and better absorbency, bleaching efficiency and whiteness index of bleached fabric which have been studied in this work.

Keywords: Pretreatment; Chemical concentration; Desizing; Scouring; Bleaching; Absorbency; Whiteness-index

\section{Introduction}

The textile dyeing sector uses large quantities of chemical companies operating challenges this project to identify the different types of chemical used and the overall quantities involved in each case; check the use of standard chemical doses in pretreatment process and check the RFD (Ready for Dyeing) material parameters i.e. absorbency, whiteness index and desizing efficiency [1]. The benefits of reducing chemical in dyeing process to reduce cost of chemical, time, water, save the energy, ultimately save the cost of pretreatment process [2].

Chemical concentration has demonstrated that substantial cost saving can be made with zero investment by improving operating practice and by changing process in chemical processing [3]. In textile processing the reducing of chemicals doses helps to ETP (Effluent Treatment Plant).

\section{Material and Method}

\section{Material}

Material used for the experiment was $100 \%$ cotton terry towel was procured from textile Park [4], Ruby dyeing II Pvt. Ltd Shirpur, Maharashtra.
A. Machine: Soft flow thies dyeing machine.

Check point of RFD material: 1. Fabric pH should be a neutral. 2 . Fabric should be free from residual peroxide \& size paste [5].

\section{Method}

Check the all parameter during RFD process of terry towel i.e.,

1. Desizing $\mathrm{pH}$

2. Bleaching $\mathrm{pH}$

3. Water $\mathrm{pH}$

4. Hardness of water

5. Hardness of bleaching water

6. Concentration of chemical: After RFD process check parameter i.e

a) $\mathrm{pH}$ of terry towel

b) Desizing efficiency

c) Absorbency 
d) Whiteness index of terry towel

\section{Experimental Work}

\section{Data collection: Lab recipe for desized white before trial}

Table 1: Study of pretreatment process before trial for desize white.

\begin{tabular}{|c|c|c|c|c|}
\hline Sr. No. & Chemical Name & $\begin{array}{c}\text { Concentration } \\
\text { of Chemical }\end{array}$ & Temperature & Time \\
\hline \multirow{3}{*}{ Step 1} & Ready scour & $0.2 \mathrm{~g} / \mathrm{l}$ & \multirow{3}{*}{$90^{\circ} \mathrm{C}$} & \multirow{3}{*}{ 20Min } \\
\hline & Acetic Acid & $0.2 \mathrm{~g} / \mathrm{l}$ & & \\
\hline & Emcozyme 40 & $0.8 \mathrm{~g} / \mathrm{l}$ & & \\
\hline \multirow{5}{*}{ Step 2} & Ready scour & $0.3 \mathrm{~g} / \mathrm{l}$ & \multirow{5}{*}{$110^{\circ} \mathrm{C}$} & \multirow{5}{*}{ 60Min } \\
\hline & Miralizer Cipha & $0.6 \mathrm{~g} / \mathrm{l}$ & & \\
\hline & Emsek ET & $1.0 \mathrm{~g} / \mathrm{l}$ & & \\
\hline & Caustic Soda Flakes & $2.0 \mathrm{~g} / \mathrm{l}$ & & \\
\hline & Hydrogen Peroxide & $10 \mathrm{~g} / \mathrm{l}$ & & \\
\hline \multirow[t]{3}{*}{ Step 3} & Miralizer Cipha & $0.1 \mathrm{~g} / \mathrm{l}$ & \multirow{3}{*}{$110^{\circ} \mathrm{C}$} & \multirow{3}{*}{ 30Min } \\
\hline & Caustic Soda Flakes & $0.5 \mathrm{~g} / \mathrm{l}$ & & \\
\hline & Hydrogen Peroxide & $2.5 \mathrm{~g} / \mathrm{l}$ & & \\
\hline \multirow[t]{2}{*}{ Step 4} & \multicolumn{2}{|c|}{ Hot wash with water } & $80^{\circ} \mathrm{C}$ & 10Min \\
\hline & \multicolumn{2}{|c|}{ Hot wash with water } & $80^{\circ} \mathrm{C}$ & $6 \mathrm{Min}$ \\
\hline Step 5 & Eskay white BHT $115 \%$ & $0.6 \mathrm{~g} / \mathrm{l}$ & $85^{\circ} \mathrm{C}$ & 30Min \\
\hline Step 6 & Acetic Acid & $0.5 \mathrm{~g} / \mathrm{l}$ & $60^{\circ} \mathrm{C}$ & 10Min \\
\hline
\end{tabular}

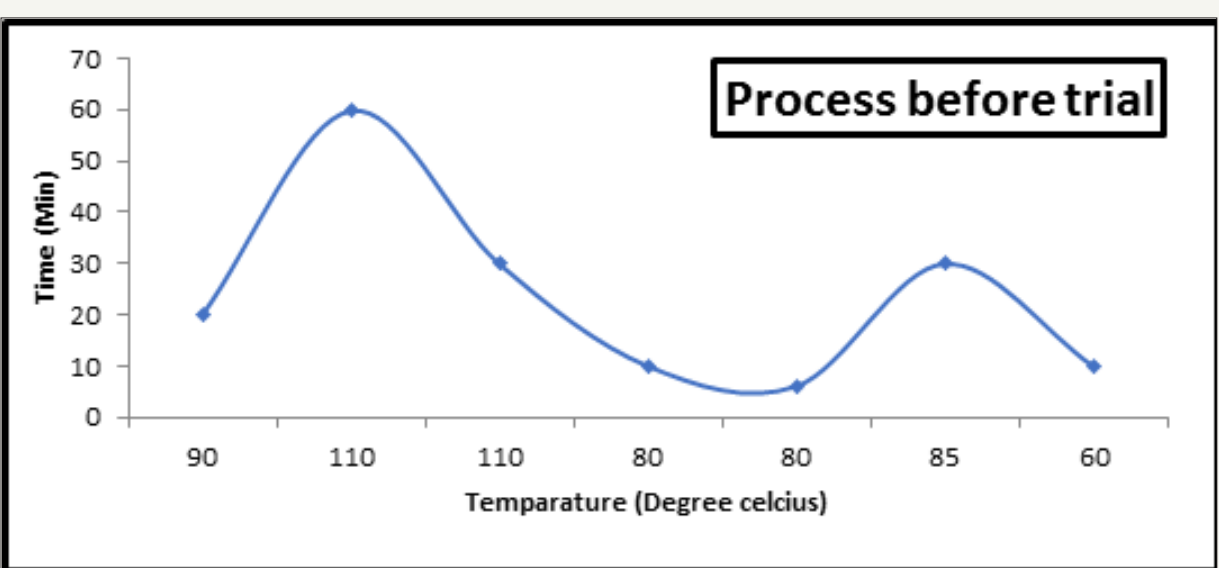

Figure 1: Pretreatment process before trial for desize white. 


\section{Data collection: Lab recipe for desized white after trial}

Table 2: Study of pretreatment process after trial.

\begin{tabular}{|c|c|c|c|c|}
\hline Sr. No. & Chemical Name & Concentration of Chemical & Temperature & Time \\
\hline \multirow{3}{*}{ Step 1} & Ready scour & $0.2 \mathrm{~g} / \mathrm{l}$ & \multirow{3}{*}{$90^{\circ} \mathrm{C}$} & \multirow{3}{*}{ 20Min } \\
\hline & Acetic Acid & $0.2 \mathrm{~g} / \mathrm{l}$ & & \\
\hline & Emcozyme 40 & $0.8 \mathrm{~g} / \mathrm{l}$ & & \\
\hline \multirow{6}{*}{ Step 2} & Ready scour & $0.3 \mathrm{~g} / \mathrm{l}$ & \multirow{6}{*}{$110^{\circ} \mathrm{C}$} & \multirow{6}{*}{ 75Min } \\
\hline & Miralizer cipha & $0.6 \mathrm{~g} / \mathrm{l}$ & & \\
\hline & Emsek ET & $1.0 \mathrm{~g} / \mathrm{l}$ & & \\
\hline & Miralizer cipha & $0.1 \mathrm{~g} / \mathrm{l}$ & & \\
\hline & Caustic soda flakes & $2.0 \mathrm{~g} / \mathrm{l}$ & & \\
\hline & Hydrogen peroxide & $10.0 \mathrm{~g} / \mathrm{l}$ & & \\
\hline \multirow{2}{*}{ Step 3} & \multicolumn{2}{|c|}{ Hot wash with water } & $80^{\circ} \mathrm{C}$ & $10 \mathrm{Min}$ \\
\hline & \multicolumn{2}{|c|}{ Hot wash with water } & $80^{\circ} \mathrm{C}$ & $6 \mathrm{Min}$ \\
\hline Step 4 & Eskay white BHT 115\% & $0.6 \%$ & $85^{\circ} \mathrm{C}$ & 30Min \\
\hline Step 5 & Acetic Acid & $0.5 \mathrm{~g} / \mathrm{l}$ & $60^{\circ} \mathrm{C}$ & 10Min \\
\hline
\end{tabular}

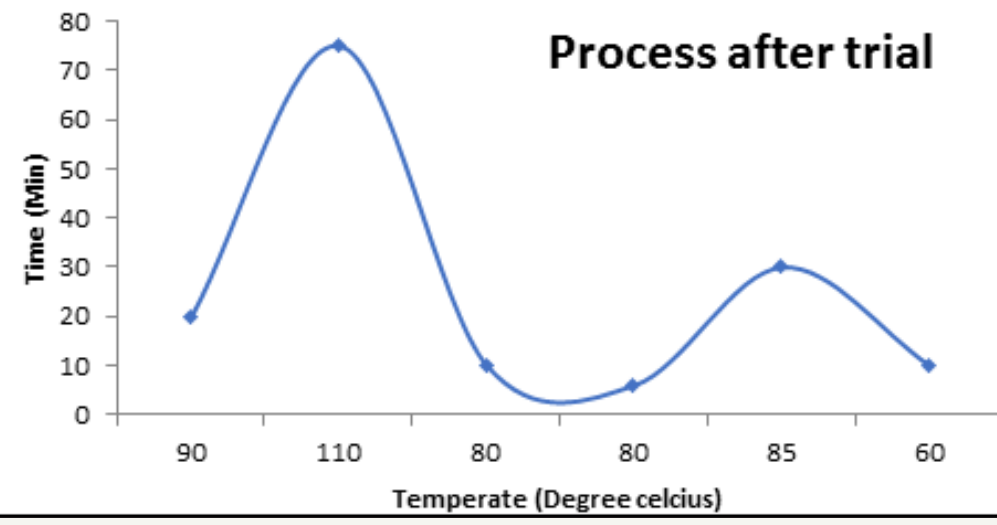

Figure 2: Pretreatment process after trial for desize white.

\section{Data collection: Bulkrecipe for desized white before trial}

Shade: White

Table 3: Study of pretreatment process before trial in bulk recipe.

\begin{tabular}{|c|c|c|c|c|c|}
\hline \multirow{2}{*}{ Sr. No. } & Check Points & Lot No. 9236-4 & $\begin{array}{c}\text { Lot No. 9266-1, } \\
\mathbf{2 , 3}\end{array}$ & Lot No. 9242-1, 2 & Lot No. 9262-2, 3 \\
\hline 1 & Water pH & 7.5 & 7.5 & 7.5 & 7.5 \\
\hline 2 & Hardness of water & 70 & 70 & 70 & 70 \\
\hline 3 & Desizing pH & 5.5 & 5.5 & 5.5 & 5.5 \\
\hline 4 & $1^{\text {st }}$ Bleaching pH & 11 & 11 & 11 & 11 \\
\hline 5 & $2^{\text {nd }}$ Bleaching pH & 11 & 11 & 11 & 70 \\
\hline 6 & Bleaching Hardness & 70 & 70 & 70 & 7.5 \\
\hline 7 & OBA pH & 9.5 & 9.5 & 9.5 & 9.5 \\
\hline 8 & Neutralization $\mathrm{pH}$ & 6.5 & 6.5 & 6.5 & 6.5 \\
\hline
\end{tabular}




\begin{tabular}{|c|c|c|c|c|c|}
\hline 9 & Whiteness index of $1^{\text {st }}$ bleached sample & 74.97 & 77.43 & 3.25 & 73.59 \\
\hline 10 & Whiteness index of $2^{\text {nd }}$ bleached sample & 74.17 & 78.31 & 73.17 & 72.49 \\
\hline 11 & Absorbency $\%$ & 72 & 72 & 70 & 50 \\
\hline 12 & GSM of towel & 595 & 700 & 613 & 546 \\
\hline 13 & Absorbency of final sample & 152.14 & 148.42 & 147.84 & 142.96 \\
\hline
\end{tabular}

\section{Data collection: Bulk recipe for desized white after trial}

Shade: White

Table 4: Study of pretreatment process after trial in bulk recipe.

\begin{tabular}{|c|c|c|c|c|c|}
\hline Sr. No. & Check Points & Lot No. 9366 & Lot No. 9369 & $\begin{array}{c}\text { Lot No. 9373-1 \& 9374-3, } \\
4\end{array}$ & Lot No. 9427-2, 3 \\
\hline 1 & Water $\mathrm{pH}$ & 7.5 & 7.5 & 7.5 & 7.5 \\
\hline 2 & Hardness of water & 70 & 70 & 70 & 70 \\
\hline 3 & Desizing $\mathrm{pH}$ & 5.5 & 5.5 & 5.5 & 5.5 \\
\hline 4 & Bleaching $\mathrm{pH}$ & 11 & 11 & 11 & 11 \\
\hline 5 & Bleaching Hardness & 70 & 70 & 70 & 70 \\
\hline 6 & OBA pH & 9.5 & 9.5 & 9.5 & 9.5 \\
\hline 7 & Neutralization $\mathrm{pH}$ & 6.5 & 6.5 & 6.5 & 6.5 \\
\hline 8 & Whiteness index of bleached sample & 77.83 & 79.55 & 77.54 & 79.11 \\
\hline 9 & Absorbency \% & 64 & 76 & 80 & 58 \\
\hline 10 & GSM of towel & 546 & 566 & 570 & 493 \\
\hline
\end{tabular}

\section{Data collection-2}

Study of RFD process in lab (2.5gpl caustic):

Table 5:

\begin{tabular}{|c|c|c|c|c|}
\hline Sr. No. & Chemical Name & Concentration of Chemical & Temperature & Time \\
\hline \multirow{3}{*}{ Step 1} & Ready scour & $0.2 \mathrm{~g} / \mathrm{l}$ & \multirow{3}{*}{$90^{\circ} \mathrm{C}$} & \multirow{3}{*}{ 20Min } \\
\hline & Acetic Acid & $0.2 \mathrm{~g} / \mathrm{l}$ & & \\
\hline & Emcozyme 40 & $0.8 \mathrm{~g} / \mathrm{l}$ & & \\
\hline \multirow{5}{*}{ Step 2} & Ready scour & $0.3 \mathrm{~g} / \mathrm{l}$ & \multirow{5}{*}{$110^{\circ} \mathrm{C}$} & \multirow{5}{*}{3 Min } \\
\hline & Miralizer Cipha & $0.6 \mathrm{~g} / \mathrm{l}$ & & \\
\hline & Emsek ET & $1.0 \mathrm{~g} / \mathrm{l}$ & & \\
\hline & Caustic Soda Flakes & $2.5 \mathrm{~g} / \mathrm{l}$ & & \\
\hline & Hydrogen Peroxide & $2.5 \mathrm{~g} / \mathrm{l}$ & & \\
\hline Step 4 & Acetic Acid & $1.2 \mathrm{~g} / \mathrm{l}$ & $55^{\circ} \mathrm{C}$ & 6Min \\
\hline Step 5 & Emcozyme Quencher M & $0.4 \mathrm{~g} / \mathrm{l}$ & $55^{\circ} \mathrm{C}$ & 10Min \\
\hline Step 6 & Miragen MER & $0.5 \mathrm{~g} / \mathrm{l}$ & $60^{\circ} \mathrm{C}$ & 5 Min \\
\hline
\end{tabular}

A. RFD Process in lab (1.5gpl caustic):

Table 6:

\begin{tabular}{|c|c|c|c|c|}
\hline Sr. No. & Chemical Name & Concentration of Chemical & Temperature & Time \\
\hline \multirow{3}{*}{ Step 1} & Ready scour & $0.2 \mathrm{~g} / \mathrm{l}$ & \multirow{3}{*}{$90^{\circ} \mathrm{C}$} & \multirow{3}{*}{ 20Min } \\
\hline & Acetic Acid & $0.2 \mathrm{~g} / \mathrm{l}$ & & \\
\hline & Emcozyme 40 & $0.8 \mathrm{~g} / \mathrm{l}$ & & \\
\hline
\end{tabular}




\begin{tabular}{|c|c|c|c|c|}
\hline \multirow{5}{*}{ Step 2} & Ready scour & $0.3 \mathrm{~g} / \mathrm{l}$ & \multirow{5}{*}{$110^{\circ} \mathrm{C}$} & \multirow{5}{*}{ 30Min } \\
\hline & MiralizerCipha & $0.6 \mathrm{~g} / \mathrm{l}$ & & \\
\hline & Emsek ET & $1.0 \mathrm{~g} / \mathrm{l}$ & & \\
\hline & Caustic Soda Flakes & $1.5 \mathrm{~g} / \mathrm{l}$ & & \\
\hline & Hydrogen Peroxide & $2.5 \mathrm{~g} / \mathrm{l}$ & & \\
\hline Step 4 & Acetic Acid & $1.2 \mathrm{~g} / \mathrm{l}$ & $55^{\circ} \mathrm{C}$ & $6 \mathrm{Min}$ \\
\hline Step 5 & Emcozyme Quencher M & $0.4 \mathrm{~g} / \mathrm{l}$ & $55^{\circ} \mathrm{C}$ & 10Min \\
\hline Step 6 & Miragen MER & $0.5 \mathrm{~g} / \mathrm{l}$ & $60^{\circ} \mathrm{C}$ & 5 Min \\
\hline
\end{tabular}

B. Before result in lab:

Table 7:

\begin{tabular}{|c|c|c|c|c|c|}
\hline \multirow[t]{2}{*}{ Sr. No. } & \multicolumn{3}{|c|}{ 2.5gpl Result in Caustic } & \multicolumn{2}{|c|}{ 1.5gpl Result in Caustic } \\
\hline & Check point & $1^{\text {st }}$ trail & $1^{\text {st }}$ trail & $2^{\text {nd }}$ trail & $2^{\text {nd }}$ trail \\
\hline 1 & Water pH & 7.5 & 7.5 & 7.5 & 7.5 \\
\hline 2 & Hardness of water & 70 & 70 & 70 & 70 \\
\hline 3 & Desizing $\mathrm{pH}$ & 5.5 & 5.5 & 5.5 & 5.5 \\
\hline 4 & Bleaching $\mathrm{pH}$ & 11 & 11 & 11 & 11 \\
\hline 5 & Peroxide killer & - & - & - & - \\
\hline 6 & Netralization $\mathrm{p}^{\mathrm{H}}$ & 6.5 & 6.5 & 6.5 & 6.5 \\
\hline 7 & Levelling $\mathrm{p}^{\mathrm{H}}$ & 6.5 & 6.5 & 6.5 & 6.5 \\
\hline 8 & Absorbency \% & 56 & 70 & 58 & 69 \\
\hline 9 & Whiteness & 70.56 & 62.87 & 62.60 & 62.87 \\
\hline 10 & GSM & 550 & 546 & 552 & 545 \\
\hline
\end{tabular}

Study of RFD process before trial in bulk processs:

Table 8:

\begin{tabular}{|c|c|c|c|c|}
\hline Sr. No. & Chemical Name & Concentration of Chemical & Temperature & Time \\
\hline \multirow{3}{*}{ Step 1} & Ready scour & $0.2 \mathrm{~g} / \mathrm{l}$ & \multirow{3}{*}{$90^{\circ} \mathrm{C}$} & \multirow{3}{*}{ 20Min } \\
\hline & Acetic Acid & $0.2 \mathrm{~g} / \mathrm{l}$ & & \\
\hline & Emcozyme 40 & $0.8 \mathrm{~g} / \mathrm{l}$ & & \\
\hline \multirow{5}{*}{ Step 2} & Ready scour & $0.3 \mathrm{~g} / \mathrm{l}$ & \multirow{5}{*}{$110^{\circ} \mathrm{C}$} & \multirow{5}{*}{ 30Min } \\
\hline & Miralizer cipha & $0.6 \mathrm{~g} / \mathrm{l}$ & & \\
\hline & Emsek ET & $1.0 \mathrm{~g} / \mathrm{l}$ & & \\
\hline & Caustic Soda Flakes & $2.5 \mathrm{~g} / \mathrm{l}$ & & \\
\hline & Hydrogen Peroxide & $2.5 \mathrm{~g} / \mathrm{l}$ & & \\
\hline Step 4 & Acetic Acid & $1.2 \mathrm{~g} / \mathrm{l}$ & $55^{\circ} \mathrm{C}$ & $6 \mathrm{Min}$ \\
\hline Step 5 & Emcozyme Quencher M & $0.4 \mathrm{~g} / \mathrm{l}$ & $55^{\circ} \mathrm{C}$ & 10Min \\
\hline Step 6 & Miragen MER & $0.5 \mathrm{~g} / \mathrm{l}$ & $60{ }^{\circ} \mathrm{C}$ & $5 \mathrm{Min}$ \\
\hline
\end{tabular}

A. Bulk RFD process before trial data: 
Table 9:

\begin{tabular}{|c|c|c|c|c|c|}
\hline & Shade & Dark Blue & Stone & Light Blue & Purple \\
\hline & Lot No. & $9179-1$ & $9316-1,2$ & $9357-1,2$ & $9583-1,2$ \\
\hline Sr. No. & \multicolumn{5}{|c|}{ Check points } \\
\hline 1 & Water pH & 7.5 & 7.5 & 7.5 & 7.5 \\
\hline 2 & Hardness of water & 70 & 70 & 70 & 70 \\
\hline 3 & Desizing $\mathrm{pH}$ & 5.5 & 5.5 & 5.5 & 5.5 \\
\hline 4 & Bleaching $\mathrm{pH}$ & 11 & 11 & 11 & 11 \\
\hline 5 & Neutralization $\mathrm{pH}$ & 6.5 & 6.5 & 6.5 & 6.5 \\
\hline 6 & Peroxide Killer & $0 \mathrm{mg} /$ Lit & $0 \mathrm{mg} / \mathrm{Lit}$ & 0mg/Lit & 0mg/Lit \\
\hline 7 & Levelling $\mathrm{pH}$ & 6.5 & 6.5 & 6.5 & 6.5 \\
\hline 8 & Absorbency \% & 50 & 70 & 48 & 50 \\
\hline 9 & Whiteness & 59.89 & 62.13 & 51.82 & 62.15 \\
\hline 10 & GSM & 406 & 558 & 560 & 553 \\
\hline 11 & Final sample Absorbency \% & 66 & 82 & 82 & 83 \\
\hline
\end{tabular}

B. Study of RFD process after trial:

Table 10:

\begin{tabular}{|c|c|c|c|c|}
\hline Sr. No. & Chemical Name & Concentration of Chemical & Temperature & Time \\
\hline \multirow{3}{*}{ Step 1} & Ready scour & $0.2 \mathrm{~g} / \mathrm{l}$ & \multirow{3}{*}{$90^{\circ} \mathrm{C}$} & \multirow{3}{*}{$20 \mathrm{Min}$} \\
\hline & Acetic Acid & $0.2 \mathrm{~g} / \mathrm{l}$ & & \\
\hline & Emcozyme 40 & $0.8 \mathrm{~g} / \mathrm{l}$ & & \\
\hline \multirow{5}{*}{ Step 2} & Ready scour & $0.3 \mathrm{~g} / \mathrm{l}$ & \multirow{5}{*}{$110^{\circ} \mathrm{C}$} & \multirow{5}{*}{ 30Min } \\
\hline & Miralizer cipha & $0.6 \mathrm{~g} / \mathrm{l}$ & & \\
\hline & Emsek ET & $1.0 \mathrm{~g} / \mathrm{l}$ & & \\
\hline & Caustic Soda Flakes & $2.0 \mathrm{~g} / \mathrm{l}$ & & \\
\hline & Hydrogen Peroxide & $2.5 \mathrm{~g} / 1$ & & \\
\hline Step 4 & Acetic Acid & $1.2 \mathrm{~g} / \mathrm{l}$ & $55^{\circ} \mathrm{C}$ & 6Min \\
\hline Step 5 & Emcozyme Quencher M & $0.4 \mathrm{~g} / \mathrm{l}$ & $55^{\circ} \mathrm{C}$ & 10Min \\
\hline Step 6 & Miragen MER & $0.5 \mathrm{~g} / \mathrm{l}$ & $60^{\circ} \mathrm{C}$ & $5 \mathrm{Min}$ \\
\hline
\end{tabular}

Part 1: Trail with 2.0 GPL caustic in RFD process: 
Table 11:

\begin{tabular}{|c|c|c|}
\hline \multicolumn{2}{|c|}{ Shade } & Navy \\
\hline \multicolumn{2}{|c|}{ Lot No. } & $9305-1$ \\
\hline Sr. No. & Check points & \\
\hline 1 & Water pH & 7.5 \\
\hline 2 & Hardness of water & 125 \\
\hline 3 & Desizing pH & 5.5 \\
\hline
\end{tabular}

\begin{tabular}{|c|c|c|}
\hline 4 & Bleaching $\mathrm{pH}$ & 11 \\
\hline 5 & Neutralization $\mathrm{pH}$ & 6.5 \\
\hline 6 & Peroxide Killer & $0 \mathrm{mg} /$ Lit \\
\hline 7 & Levelling $\mathrm{pH}$ & 6.5 \\
\hline 8 & Absorbency $\%$ & 78 \\
\hline 9 & Whiteness & 58.64 \\
\hline 10 & GSM & 429 \\
\hline 11 & Final sample Absorbency $\%$ & 58 \\
\hline
\end{tabular}

A. After trail results: 2 GPL Caustic (Bulk Trial):

Table 12:

\begin{tabular}{|c|c|c|c|c|}
\hline Sr. No. & Chemical Name & Concentration of Chemical & Temperature & Time \\
\hline \multirow{3}{*}{ Step 1} & Ready scour & $0.2 \mathrm{~g} / \mathrm{l}$ & \multirow{3}{*}{$90^{\circ} \mathrm{C}$} & \multirow{3}{*}{ 20Min } \\
\hline & Acetic Acid & $0.2 \mathrm{~g} / \mathrm{l}$ & & \\
\hline & Emcozyme 40 & $0 . \mathrm{g} / \mathrm{l}$ & & \\
\hline \multirow{5}{*}{ Step 2} & Ready scour & $0.3 \mathrm{~g} / \mathrm{l}$ & \multirow{5}{*}{$110^{\circ} \mathrm{C}$} & \multirow{5}{*}{30 Min } \\
\hline & Miralizer cipha & $0.6 \mathrm{~g} / \mathrm{l}$ & & \\
\hline & Emsek ET & $1.0 \mathrm{~g} / \mathrm{l}$ & & \\
\hline & Caustic Soda Flakes & $2.5 \mathrm{~g} / \mathrm{l}$ & & \\
\hline & Hydrogen Peroxide & $2.5 \mathrm{~g} / \mathrm{l}$ & & \\
\hline Step 4 & Acetic Acid & $1.2 \mathrm{~g} / \mathrm{l}$ & $55^{\circ} \mathrm{C}$ & 6 Min \\
\hline Step 5 & Emcozyme Quencher M & $0.4 \mathrm{~g} / \mathrm{l}$ & $55^{\circ} \mathrm{C}$ & 10Min \\
\hline Step 6 & Miragen MER & $0.5 \mathrm{~g} / \mathrm{l}$ & $60^{\circ} \mathrm{C}$ & $5 \mathrm{Min}$ \\
\hline
\end{tabular}

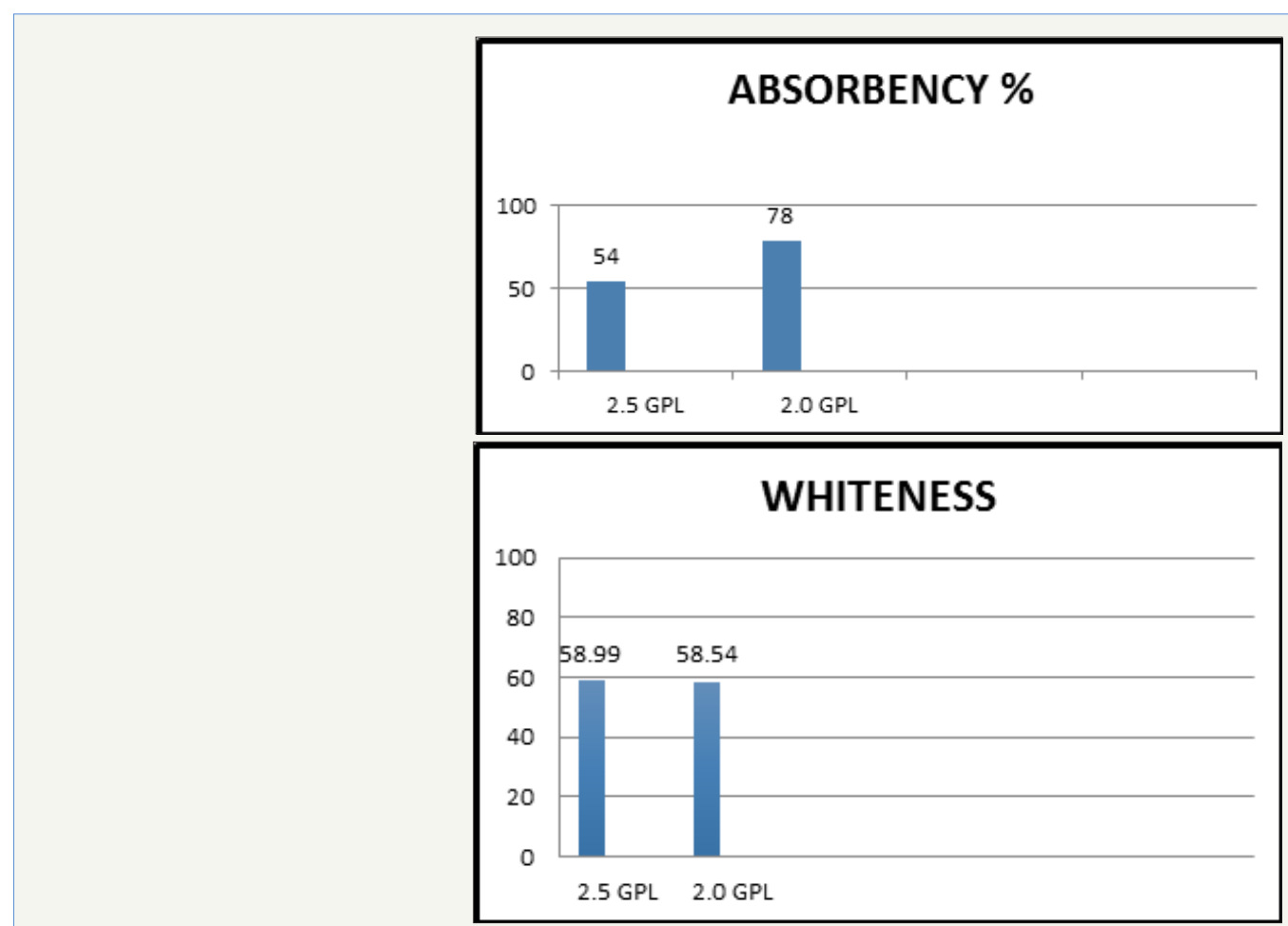

Figure 3: 
Part 2: Trail with 2.5 GPL caustic in RFD process:

Table 13:

\begin{tabular}{|c|c|c|c|c|}
\hline Sr. No. & Chemical Name & Concentration of Chemical & Temperature & Time \\
\hline \multirow{3}{*}{ Step 1} & Ready scour & $0.2 \mathrm{~g} / \mathrm{l}$ & \multirow{3}{*}{$90^{\circ} \mathrm{C}$} & \multirow{3}{*}{ 20Min } \\
\hline & Acetic Acid & $0.2 \mathrm{~g} / \mathrm{l}$ & & \\
\hline & Emcozyme 40 & $0.8 \mathrm{~g} / \mathrm{l}$ & & \\
\hline \multirow{5}{*}{ Step 2} & Ready scour & $0.3 \mathrm{~g} / \mathrm{l}$ & \multirow{5}{*}{$110^{\circ} \mathrm{C}$} & \multirow{5}{*}{ 30Min } \\
\hline & Miralizer cipha & $0.6 \mathrm{~g} / \mathrm{l}$ & & \\
\hline & Emsek ET & $1.0 \mathrm{~g} / \mathrm{l}$ & & \\
\hline & Caustic Soda Flakes & $2.5 \mathrm{~g} / \mathrm{l}$ & & \\
\hline & Hydrogen Peroxide & $2.5 \mathrm{~g} / \mathrm{l}$ & & \\
\hline Step 4 & Acetic Acid & $1.2 \mathrm{~g} / \mathrm{l}$ & $55^{\circ} \mathrm{C}$ & 6Min \\
\hline Step 5 & Emcozyme Quencher M & $0.4 \mathrm{~g} / \mathrm{l}$ & $55^{\circ} \mathrm{C}$ & 10Min \\
\hline Step 6 & Miragen MER & $0.5 \mathrm{~g} / \mathrm{l}$ & $60^{\circ} \mathrm{C}$ & 5 Min \\
\hline
\end{tabular}

A. Before trail results:

Table 14:

\begin{tabular}{|c|c|c|}
\hline \multicolumn{2}{|r|}{ Shade } & Grey \\
\hline \multicolumn{2}{|r|}{ Lot No. } & $9644-1,2$ \\
\hline Sr. No. & Check points & \\
\hline 1 & Water $\mathrm{pH}$ & 7.5 \\
\hline s2 & Hardness of water & 70 \\
\hline 3 & Desizing $\mathrm{pH}$ & 5.5 \\
\hline 4 & Bleaching $\mathrm{pH}$ & 11 \\
\hline 5 & Neutralization $\mathrm{pH}$ & 6.5 \\
\hline 6 & Peroxide Killer & Nil \\
\hline 7 & Levelling $\mathrm{pH}$ & 6.5 \\
\hline 8 & Absorbency \% & 50 \\
\hline 9 & Whiteness & 61.29 \\
\hline 10 & GSM & 410 \\
\hline 11 & Final sample Absorbency \% & 40 \\
\hline
\end{tabular}

B. After trail results:
Table 15:

\begin{tabular}{|c|c|c|}
\hline \multicolumn{2}{|r|}{ Shade } & \multirow{2}{*}{$\begin{array}{c}\text { Brunt Orange } \\
9645-3\end{array}$} \\
\hline & Lot No. & \\
\hline Sr. No. & Check points & \\
\hline 1 & Water $\mathrm{pH}$ & 7.5 \\
\hline 2 & Hardness of water & 70 \\
\hline 3 & Desizing $\mathrm{pH}$ & 5.5 \\
\hline 4 & Bleaching $\mathrm{pH}$ & 11 \\
\hline 5 & Neutralization $\mathrm{pH}$ & 6.5 \\
\hline 6 & Peroxide Killer & Nil \\
\hline 7 & Levelling $\mathrm{pH}$ & 6.5 \\
\hline 8 & Absorbency \% & 50 \\
\hline 9 & Whiteness & 67.02 \\
\hline s10 & GSM & 408 \\
\hline 11 & Final sample Absorbency \% & 40 \\
\hline
\end{tabular}


Results and Discussion

Graphical representation

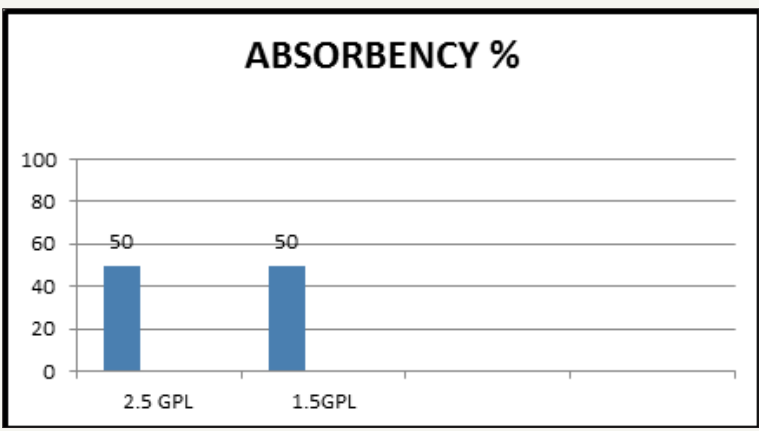

WHITENESS

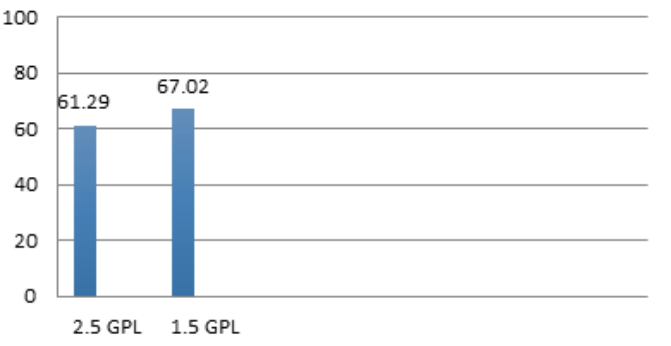

Figure 4:

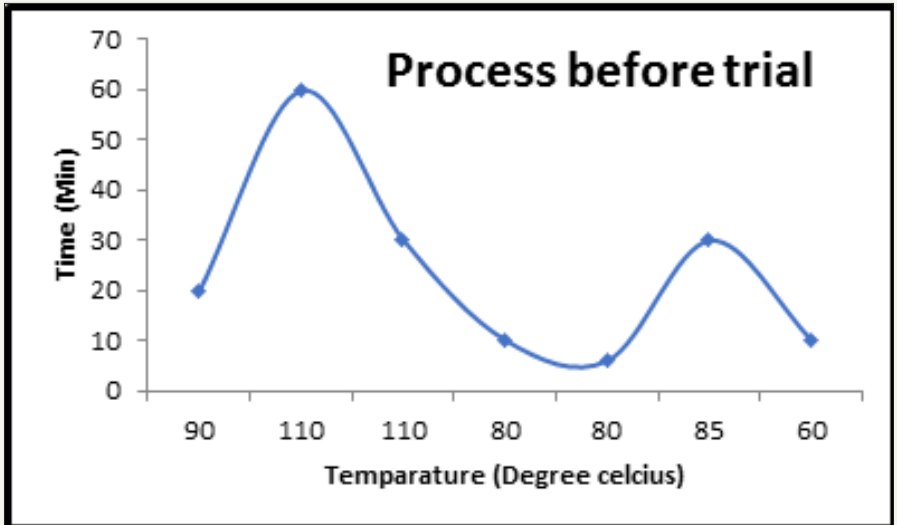

Figure 5: Process for white before trail.

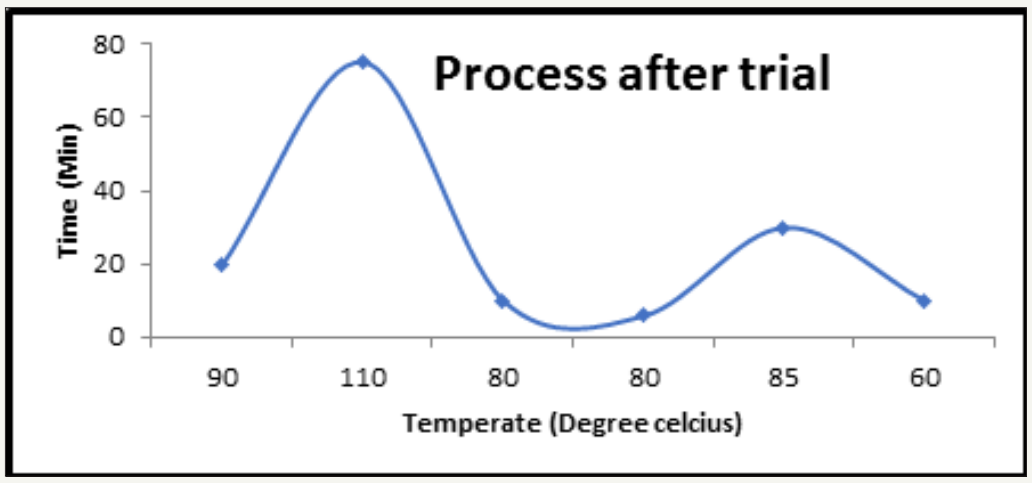

Figure 6: Process for white after trail. 


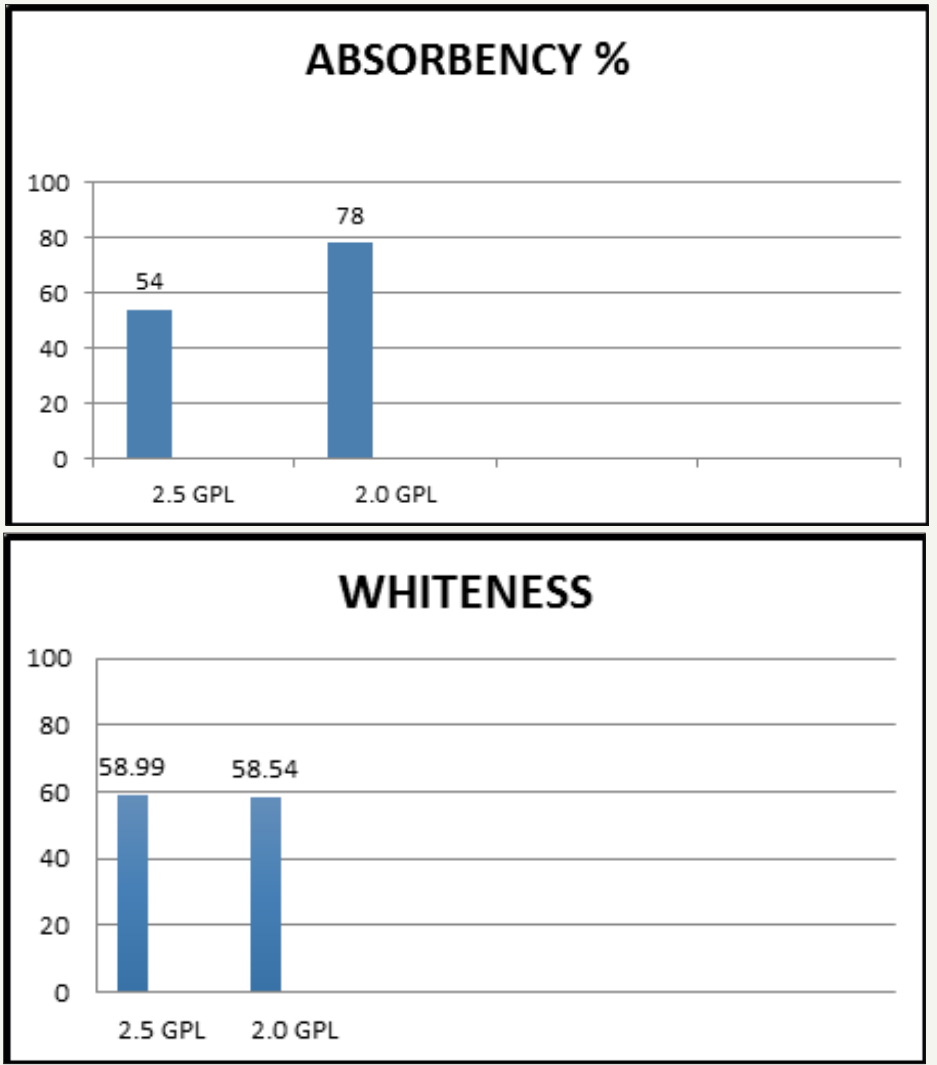

Figure 7: Bulk RFD process before trial.

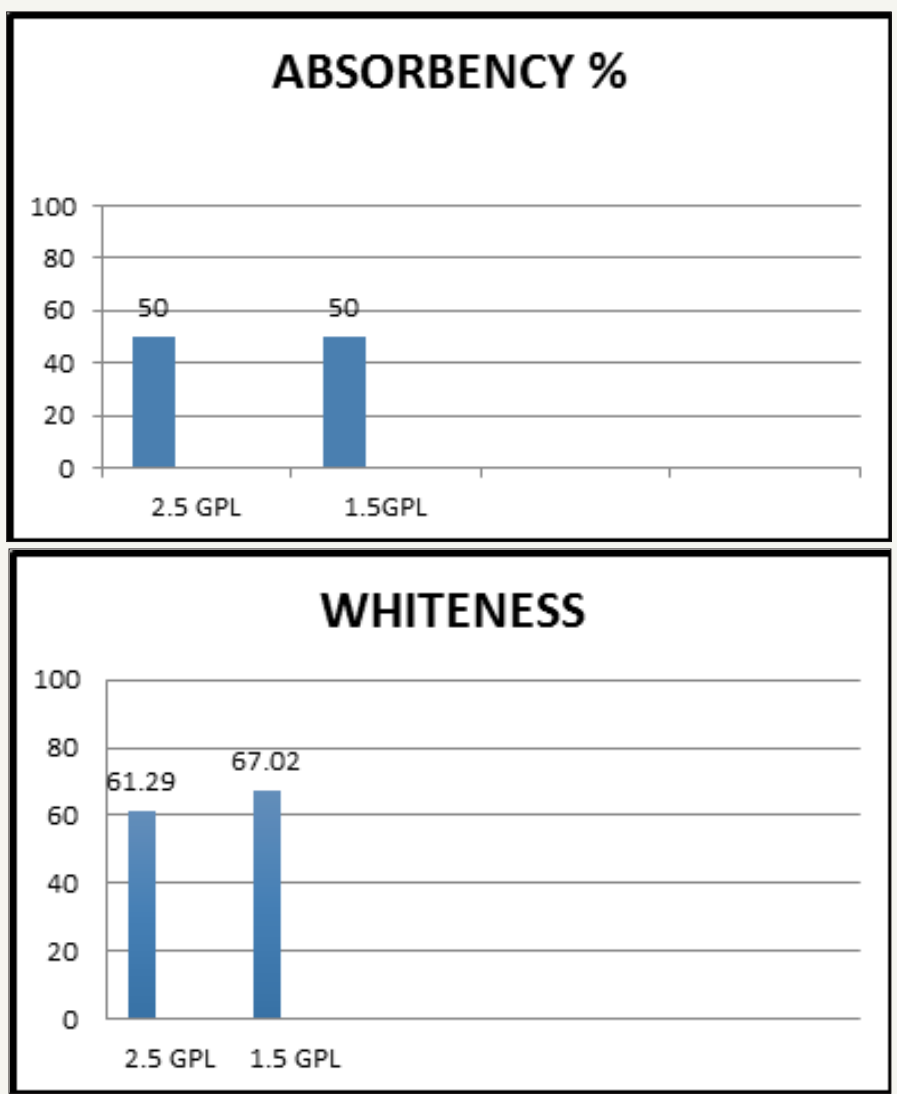

Figure 8: Bulk RFD process after trail. 


\section{Discussion}

In this project we have try to reduce the chemical consumption in pretreatment of terry towel for this work we first study standard dosages of each chemical used in pretreatment and dyeing of terry towel [6]. After this for month we study the result of these $\mathrm{pH}$ and dosages in pretreatment of terry towel and check parameter like absorbency, whiteness, pH desizing efficiency [7-10]. The discusses this with our mentor and other shift officer then with their help we reduce some quantity of chemical from standard dosges from of caustic and take trial in lab and compair it' result against result of standard dosages of caustic and then again under their guidance take trail in bulk production of terry towel by reducing the standard dosges of chemical used in pretreatment and with constant $\mathrm{pH}$, time and temperature and compare result this dosages against standard dosage [11]. In other hand we also study the process of wnite shade in bulk in which bleaching process is carried out two time at $110{ }^{\circ} \mathrm{C}$ for $90 \mathrm{~min}$ and $110{ }^{\circ} \mathrm{C}$ for $75 \mathrm{~min}$. We reduce one process of them.

\section{Conclusion}

In this study, from our experimental result we concluded that desizing efficiency, whiteness index, and absorbency of terry towel is good. The result which is observed and analyze is giving similar data as work which is performed in industry [12]. This study is perform with the use of $2.5,2$ and $1.5 \mathrm{~g} / \mathrm{l} \mathrm{NaOH}$ in pretreatment process and compared with $2.5 \mathrm{~g} / \mathrm{l}$ standard concentration of $\mathrm{NaOH}$ which is a conventional process. From our experimental result we can implement the use of $2 \mathrm{~g} / \mathrm{l}$ and $1.5 \mathrm{~g} / \mathrm{l}$ caustic in pretreatment process of terry towel instead of standard dosage i.e., $2.5 \mathrm{~g} / \mathrm{l}$. This is the following points which are obtained as a result after above studies during project work:

1. This process minimizes the process of two-time bleaching process for white shade by changing the time of bleaching process at $110^{\circ} \mathrm{C}$ for $75 \mathrm{~min}$.

2. Reduction in the chemical consumption i.e $\mathrm{NaOH}$, Hydrogen peroxide and Peroxide Stabilizer in desizing, scouring, and bleaching process of fabric is obtained $[13,14]$

3. Saving of time, water, temperature, and electricity due to less time-consuming process.

4. Reduces the ETP load by using less concentration of chemical, and due to less $\mathrm{NaOH}$ concentration the effluent water become less alkaline and as a result the less amount of acid is using to neutralize effluent water.

5. Reduce the cost of pretreatment process of terry towel due to less chemical usage.

Thus, by above points we can say that this research work is giving good approach of using optimized quantity of chemical and auxiliaries in pretreatment process of cotton fabric.

\section{References}

1. Rathi AKA, Bhanujan KB (2004) Cleaner technology development- some practical steps for developing countries. Journal of Environmental Science \& Engineering 46(4): 257.

2. Chi IT, Yi ling Y, Ting Chien C, Chi Jen C (2011) The optimal operational design for a three-effect vacuum evaporator and an energy performance assessment. International Journal of Energy Science 1(2): 119.

3. George C, Cushnie (2009) Pollution prevention and control technologies for plating operations. CIA Resources Inc., USA, p. 4.

4. Muhammad Ayaz Shaikh (2009) Environmental issues related with textile sector. Pakistan Trade Journal, Pakistan, p. 36.

5. Report of World Textile Chemicals Industry (2008) Industry study with forecasts for 2012 \& 2017. pp. 3-4.

6. (1995) Standard methods for the examination of water and wastewater ( $19^{\text {th }}$ edn), Published Jointly by American Public Health Association, American Water Works Association and Water Environment Federation, Washington DC, USA, pp. 1-24.

7. Textile Exchange Online (2009) Recent market trends in the textile chemical industry. pp. 1-2.

8. Wastewater Sampling (2013) SESD operating procedure No. SESDPRO306-R3, USEPA, USA. pp. 14

9. (2009) Water \& wastewater-methods of sampling and analysis, IS-30251989 Reaffirmed 2008: HPPWD schedule of rates.

10.(2011) Water and wastewater analysis-A guide manual by central pollution control board.

11. Shenai VA (1991) Technology of bleaching and mercerizing. Sevak Publications ( $2^{\text {nd }}$ edn.), New Delhi, India.

12. Shore J (2002) Colourant \& auxiliaries. Woodhead Publishing Limited, Hampshire, England, 2: 602-607.

13. Savita P, Tushar AS, Madhuri K, Sujit G, Raichurkar PP. International Dyer \& Textile Finisher 4(4): 42-45.

14. Shahrukh D, Tushar AS, Raichurkar PP (2018) Colorage 5: 35-42.
Creative Commons Attribution 4.0

International License

For possible submissions Click Here

\section{Submit Article}

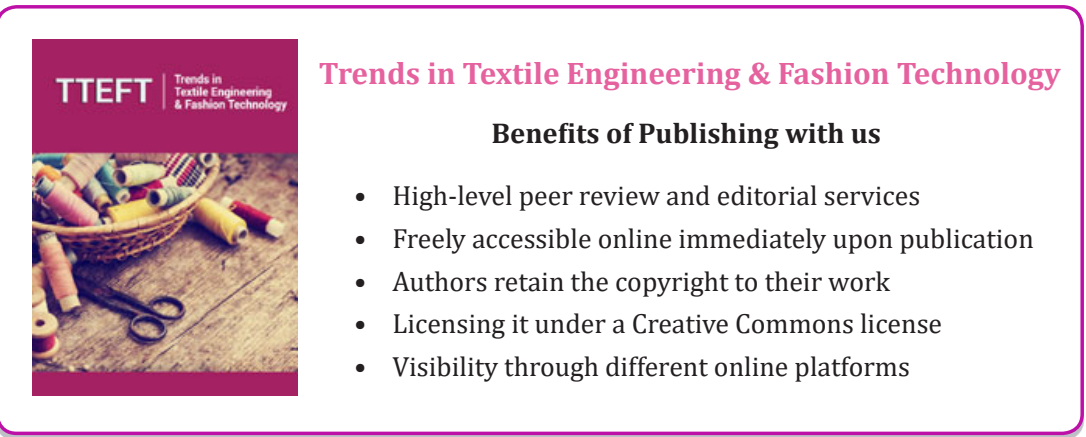

